\title{
Asie orientale : le retour du politique
}

Jean-Luc Domenach

\section{(2) OpenEdition}

Journals

Édition électronique

URL : http://journals.openedition.org/conflits/253

DOI : $10.4000 /$ conflits.253

ISSN : 1777-5345

Éditeur :

CCLS - Centre d'études sur les conflits lilberté et sécurité, L'Harmattan

Édition imprimée

Date de publication : 15 mai 1996

ISSN : 1157-996X

Référence électronique

Jean-Luc Domenach, «Asie orientale : le retour du politique », Cultures \& Conflits [En ligne], 21-22 |

printemps-été 1996, mis en ligne le 15 mars 2006, consulté le 30 mars 2021. URL : http://

journals.openedition.org/conflits/253; DOI : https://doi.org/10.4000/conflits.253

Ce document a été généré automatiquement le 30 mars 2021.

Creative Commons License 


\title{
Asie orientale : le retour $\mathrm{du}$ politique
}

\author{
Jean-Luc Domenach
}

1 Quand on aborde la question de la régionalisation de l'Asie orientale, on ne peut pas éviter de rappeler brièvement l'énorme différence entre ce processus et celui qui travaille l'Europe et l'Amérique du Nord. En effet, la régionalisation de l'Asie orientale n'est pas le résultat d'une stratégie concertée des États et elle n'a pas été, à ce jour, conclue par une négociation politique ou commerciale. Au contraire, c'est un processus principalement économique qui se développe sans objectifs et sans calendrier explicites, et qui ne possède aucune traduction institutionnelle précise, puisque les associations régionales existantes recouvrent beaucoup moins (ASEAN/AFTA) ou beaucoup plus (APEC) d'espace que celui qui est affecté pour le processus de régionalisation en Asie orientale ${ }^{1}$. La question qui se pose d'emblée est donc celle de savoir quel rôle reste dévolu aux États et plus généralement au politique dans une région où des forces économiques très puissantes paraissent libérées de leur contrôle. Et la tentation devient forte d'imaginer que l'Asie orientale est en passe de se libérer du fardeau de la politique pour s'abandonner tout entière aux flux de l'économie et des autres phénomènes transnationaux. Cette tentation nous paraît dangereuse, parce qu'elle exagère des faits importants, mais non dominants. Il nous semble en effet que les États ont collaboré à la régionalisation et qu'ils l'ont contrôlée de façon à ne consentir aucun abandon de souveraineté essentiel; que le remodelage des espaces régionaux n'apporte que des nuances secondaires à l'ordre inter-étatique dans la région; et surtout que le rapprochement des économies et des sociétés prépare un retour en force du politique en Asie orientale, tant sur les scènes intérieures que sur la scène régionale ${ }^{2}$.

2 Le rôle des États Rappelons tout d'abord que la régionalisation économique de l'Asie orientale a été initialement le résultat de deux grands mouvements d'investissements, le premier japonais, et le second émanant des Nouveaux Pays Industrialisés. Au milieu des années quatre-vingt, l'enchérissement du yen à la suite des accords du Plaza a contraint l'industrie japonaise à se délocaliser. Le fait décisif est qu'une partie notable 
(mais non majoritaire) des investissements japonais ont été effectués en Asie orientale. Pourquoi? A cause des écarts de coûts et pour d'évidentes raisons de proximité géographique et culturelle. Mais la politique n'a pas été absente de ce choix. D'abord, parce que, malgré la prudence que lui inspirait le désastre de 1945, le gouvernement japonais avait continué à encourager l'établissement de liens étroits avec les pays d'Asie orientale. Des habitudes avaient été prises, des réseaux existaient, les diplomates étaient d'accord : il était politiquement légitime que les firmes japonaises investissent en Asie. En outre, le choix asiatique d'importants investisseurs japonais a probablement été favorisé par la conjoncture politique régionale. Car dans les Nouveaux pays industrialisés et chez leurs émules d'Asie, après une décennie caractérisée par la crainte d'une extension des conflits d'Indochine, les années quatre-vingt ont été celles des apaisements politiques: que l'on pense, entre autre, à la chute de Marcos aux Philippines (1986), et à la démocratisation de Taïwan et de la Corée du Sud (1987). De toute façon, l'imbrication entre les milieux d'affaires et ceux de la politique est telle, au Japon, que des investissements massifs émanant de grandes firmes privées sont impensables en dehors d'une étroite concertation avec les milieux gouvernementaux. Ceci ne signifie pas que les investissements sont déterminés par des arguments politiques, ni que la politique est commandée par les investissements : il existe une connivence entre politiques et hommes d'affaires. Mais le choix final est toujours que la politique s'efface au profit des affaires, y compris en Asie orientale où les armées japonaises ont souvent laissé un souvenir horrible. A la fin des années quatre-vingt, les Nouveaux pays industrialisés (Corée du Sud, Taïwan, Singapour), ont pris le relais des Japonais pour investir massivement en Asie. Comme au Japon, la décision est à l'origine économique : il s'agit de délocaliser des industries menacées par les surcoûts (salaires, terrains, etc.). Comme au Japon, aussi, il faut parler, non de démission, mais de connivence de l'État, bien que les situations diffèrent. Le cas le plus simple est probablement celui de Singapour, où les contrôles politiques sur les investissements sont très étroits, et où, à la fin de la décennie, le gouvernement a adopté une politique résolument " asiatiste ", à l'intérieur comme à l'extérieur. Au début de son processus de démocratisation, la Corée du Sud est un pays où l'économie reste très contrôlée par l'intermédiaire des grands groupes, les chaebols. Ses investissements en Malaisie ou au Vietnam s'inscrivent en partie dans le cadre politique de l'activisme régional d'un État divisé. L'orientation vers la Chine est facilitée, en septembre 1992, par l'établissement de relations diplomatiques. Désormais, les investissements sur le continent chinois se concentrent à Shanghaï, et surtout dans les traditionnelles zones de contiguïté coréenne du Shandong et du Nord-Est. Le cas de Taïwan fait en revanche difficulté. Car malgré les contrôles étatiques, les délocalisations décidées à la fin des années quatrevingt n'ont qu'en partie obéi aux orientations "sudistes" données par le gouvernement (Malaisie, Thaïlande, Vietnam). L'un des plus extraordinaires exemples de flux financiers clandestins est donc celui des investissements taïwanais en Chine. Leur ampleur est totalement inconnue. On sait seulement qu'elle est considérable : 2 milliards de dollars selon les autorités, 20 milliards au moins suivant les observateurs extérieurs, jusqu'à quatre fois plus d'après des chercheurs taïwanais !... Le fait est que le gouvernement de Taipeh n'a pas eu les moyens d'empêcher une vague de délocalisations vers une destination qu'il avait interdite. Les motifs immédiats de ces délocalisations en Chine étaient économiques, et en fait bien souvent affectifs, mais ils se protégeaient derrière deux principes politiques officiels : l'un, traditionnel, suivant lequel il n'existe qu'une seule Chine; et l'autre, plus récent, suivant lequel, aux yeux de 
Taipeh, la guerre civile est terminée, et les échanges « entre les deux rives " peuvent reprendre jusqu'à un certain point (d'ailleurs peu clair). L'exception taïwanaise en désigne une autre, moins nette encore : celle des Chinois de Hong Kong. A eux seuls, ceux-ci ont assuré environ la moitié des investissements dits «étrangers » en Chine populaire. Les investissements ont été réalisés, a partir du milieu des années quatrevingt, dans un objectif de délocalisation avec l'assentiment plus ou moins forcé des autorités de la colonie. En effet (et l'on retrouve ici la politique...) ils étaient, dans leur "spontanéité » même, la conséquence de l'accord sino-britannique de 1984 sur la rétrocession de Hong Kong à la Chine en 1997. Les grands tycoons de la colonie, en même temps qu'ils répondaient à des nécessités économiques, anticipaient une annexion désormais inéluctable et cherchaient à "se placer » auprès des autorités de Pékin.

Une régionalisation sous contrôle Résumons : sauf à Taïwan et dans un sens à Hong Kong, les investissements ont bénéficié d'une véritable connivence des États. Ceux-ci, par la suite, ont en général eu l'habileté de se montrer discrets, mais sans abandonner leur surveillance, et sans négliger d'intervenir chaque fois que nécessaire. Un exemple intéressant est celui du processus d'élargissement de la région est-asiatique. Il n'a pas été décidé par un vote, c'est la réalité qui a évolué, c'est-à-dire que des espaces supplémentaires se sont successivement amalgamés à l'ensemble déjà régionalisé par les investissements et le commerce. Telle est bien l'originalité du processus asiatique : on repère peu de décisions, de coupures juridiques mais des processus, des inflexions, des courbures. Toutefois, ces évolutions demeurent guidées et contrôlées par les gouvernements. L'économie constitue la substance même du processus : ce sont les investissements et le commerce. Mais la politique lui ouvre la voie. Par exemple, l'intérêt stratégique que le Japon portait à la Corée du Sud et à Taïwan au coeur même de la guerre froide a précédé et préparé le déclenchement d'une vague d'investissements. Plus récemment, la compréhension politique croissante que les gouvernements du Japon et des NPI ont manifestée au régime réformateur de Deng Xiaoping durant les années quatre-vingt a ouvert la voie à "l'asiatisation » de l'économie chinoise depuis $1989^{3}$. Encore plus récent est le cas de la Birmanie. En refusant de suivre les Occidentaux dans leur condamnation du régime militaire birman, les principaux pays asiatiques lui ont adressé, depuis plusieurs années, un message sans équivoque : ils étaient prêts (à la condition, il est vrai, de quelques aménagements de façade) à accueillir ce pays dans le grand jeu économique de la régionalisation. De leur côté, les prétoriens de Rangoon ont multiplié les signaux politiques, d'abord en direction de Pékin, puis de Singapour, de Djakarta et de Kuala Lumpur. De fait, on a l'impression que les gouvernements de l'ASEAN sont en état de fournir un « visa pour la régionalisation " plus crédible que celui de la Chine (et plus spectaculaire que celui du Japon). On notera que les pays candidats sont admis dans la région quand ils satisfont à un certain nombre de règles implicites évidemment. La première est sans doute la stabilité politique: c'est celle que M. Ramos s'emploie aujourd'hui à expliquer dans l'espoir de faciliter l'intégration économique des Philippines. La seconde est celle d'une modération de façade dans la répression des désordres et des oppositions : c'est celle que les généraux birmans ont mise en oeuvre récemment en relâchant Mme Aung San Suu Kyi. Enfin, il est une règle qui dépasse toutes les autres : celle qui consiste à jouer le jeu (c'est-à-dire à s'ouvrir aux échanges et aux investissements), mais sans gêner la liberté des autres joueurs. L'Asie orientale d'aujourd'hui est un univers foncièrement marchand, mais où chaque marchand agit suivant ses propres règles, le plus fort 
dictant chaque fois qu'il le peut sa loi au plus faible. En Europe, chaque nouveau membre s'engage à respecter des règlements communautaires. En Asie, l'engagement implicite est de respecter l'autre, dans ses intérêts, dans ses filouteries et dans ses crimes. Il entraîne, en échange, une sorte de "droit de tirage» de solidarité ou simplement de distraction dont ont profité presque tous les gouvernements : la Chine bien sûr, mais aussi la Thaïlande (pour ses exactions au Cambodge), l'Indonésie (pour ses massacres à Timor) ou Singapour (pour sa politique répressive). Encore existe-t-il des pays plus ou moins égaux que les autres. La Chine, par exemple, est presque complètement dispensée de modération policière: elle le doit tout autant à son ancienneté (en Asie, cela compte) qu'à sa masse. Le Japon se trouve dans un statut plus complexe et qui autorise des variations : à la fois grand et petit, ancien et jeune, respecté et méprisé. A l'inverse, certains comptent moins que les autres: Taïwan, malgré sa puissance économique, et parce que la Chine le veut ainsi ; ou le Vietnam, nouvel adhérent qui n'a pas encore fait oublier ses erreurs d'hier. Cette esquisse de hiérarchie ne recouvre pas celle de la puissance économique : le statut du Japon et de la Corée, par exemple, est très inférieur à leur poids économique réel. A rebours, les États d'Asie du Sud-Est ont augmenté leur influence en se groupant dans l'ASEAN et aussi (nous y reviendrons) en organisant des consultations sur les problèmes stratégiques régionaux. En tout cas, si l'on réfléchit à la formation de la "région Asie», une conclusion s'impose : celle-ci n'est certes pas le résultat exclusif, ni même principal, d'une stratégie délibérée des États. Mais elle n'est pas non plus l'effet du jeu incontrôlé des forces économiques transnationales. Elle a été orientée et contrôlée par les dirigeants politiques. En ce sens, elle est aussi une construction politique à contenu limité. Cette conclusion, cependant, souligne une difficulté analytique. S'il s'agit d'une construction politique, comment expliquer qu'elle soit si peu explicite? Nous sommes habitués à penser que tout ce qui est politique relève d'un projet et la construction européenne en est un cas d'école. Pourtant, les idéologies pan-asiatiques ont été à la fois trop anciennes (elles se sont développées de la fin du dix-neuvième siècle à la Deuxième guerre Mondiale) et, en général, trop liées au Japon pour servir de référence explicite. On ne trouve pas trace, dans les années soixante-dix ou quatre-vingt, d'une utopie ou, à défaut, d'un projet " asiatique » au Japon, en Corée ou à Taïwan, d'où est partie la régionalisation économique. C'est plus tard, à partir du début des années quatre-vingt-dix, à Singapour et en Malaisie, qu'est apparu un discours très officiel sur les «valeurs asiatiques " ${ }^{4}$. En outre, la régionalisation n'a nullement été préparée par un rapprochement spontané ou organisé entre les cultures. On serait bien en mal de trouver, en Asie, l'équivalent des rapprochements culturels qui se sont opérés au lendemain de la deuxième guerre mondiale, entre les principales sociétés européennes, en particulier d'Allemagne et de France. Par exemple, les opinions publiques du Japon, de Chine et de Corée sont restées hostiles. Et, pour ne prendre qu'un exemple, les pêcheurs thaïlandais ont dépouillé et tué des dizaines de milliers de boat people vietnamiens. A l'origine, la régionalisation de l'Asie orientale a été le fait des seules élites asiatiques. Le fait, et non le dit : encore une fois, il n'y a pas eu de grands discours ni de plates-formes programmatiques, mais une succession de choix concrets, non institutionnels, à la limite de l'économique et du politique. Une explication est que, depuis 1945, la politique japonaise a été fort peu discursive (à cause du poids des ÉtatsUnis) et de la priorité accordée à l'économie. Une autre est que, par la suite, les NPI (sans même citer Hong Kong) étaient politiquement incapables de concevoir un projet asiatique. Ces États divisés et menacés (Corée du Sud, Taïwan) ou minuscules 
(Singapour) ne possédaient pas tous les attributs d'États-nations. Enfin, chez les derniers venus dans le jeu asiatique, les égoïsmes nationaux jouent un rôle essentiel : le Vietnam, par exemple, cherche d'abord à échapper au désastre économique, et la Chine à profiter de la prospérité régionale. Mais la principale explication tient au contenu de la régionalisation de l'Asie. Si cette régionalisation s'est à l'origine développée sans projet politique, c'est que justement elle ne s'appliquait pas au domaine politique. A aucun moment il n'a été question de créer une organisation ou un système de relations qui nécessiteraient des abandons de souveraineté. Bien au contraire, une règle d'or du concert asiatique est que rien ne doit porter atteinte à l'indépendance et à la souveraineté des États. Ceux-ci sont seulement d'accord pour faire le maximum de commerce entre eux et avec le reste du monde. Encore cet assentiment doit-il demeurer aussi implicite que possible : car des discours trop clairs, trop "vocaux", donneraient une allure durable à ce qui n'est qu'une conjonction circonstancielle d'intérêts. La régionalisation de l'Asie orientale a été le résultat d'une succession de choix économiques, tous fragiles et réversibles, émanant des élites des différents pays. $\mathrm{Au}$ moins jusqu'à une période très récente, elle ne s'est donc inspirée d'aucun autre argument fort que celui de l'intérêt des entreprises et des États concernés. Cette accumulation de calculs n'a jusqu'à présent suscité aucun sentiment d'identité collective. Lorsque l'on voyage en Asie, que l'on interroge des hommes d'affaires ou que l'on fouine dans les librairies, on a bien du mal à trouver des réponses à la question essentielle de l'espace asiatique pertinent. Qu'est-ce que l'Asie ? Cette question, à la vérité, n'est guère posée en Asie même, où les sous-ensembles régionaux qui font culturellement sens sont plus étroits: Extrême-Orient confucéen, Asie du Sud-Est malaise et islamisée, Asie du Sud indianisée et où les cultures nationales sont extrêmement vivaces. S'il existe une régionalisation culturelle, c'est par une sorte d'effet dérivé : disposant de plus d'argent et d'informations, les hommes circulent plus, les sociétés s'uniformisent et se rapprochent; les images, les symboles commencent à passer de l'une à l'autre. Le phénomène n'en est qu'à ses débuts, et il ne se distingue pas toujours nettement dans les pays longtemps fermés d'un phénomène plus général d'ouverture et de mondialisation.

4 Le renforcement de l'« État riziculteur » La régionalisation n'a donc jusqu'à présent pas atteint sérieusement la souveraineté des États. Celle-ci s'est même renforcée depuis une vingtaine d'années. Tout d'abord, s'ils ont souvent su lâcher la bride aux entreprises, les appareils d'État ont tiré profit de leurs gains, par de nombreuses ponctions (et pas seulement fiscales). Ils ont donc un peu partout renforcé leurs capacités redistributives ainsi que leurs équipements militaires. L'Asie orientale occupe actuellement le premier rang au monde dans deux domaines très différents: les augmentations de salaires et les achats d'armes. En second lieu, grâce à leur renforcement financier et militaire, les États sont souvent parvenus à consolider leurs frontières et à parfaire l'unification de leur territoire. Le cas le plus spectaculaire, à cet égard, est celui de l'État birman qui, en quelques années, vient de mettre fin à la plupart des dissidences ethniques et politiques qui le déchiraient jusque là. Les États parviennent en général à réduire les effets corrosifs des ouvertures économiques. Ainsi, la constitution d'une sorte de polarité entre la Chine du Sud, Hong Kong et Taïwan, n'a pas posé de vraies difficultés aux appareils administratifs. La Chine a dédoublé sa frontière en établissant une «zone tampon » à Shenzhen. Hong Kong a multiplié ses patrouilles frontalières. Et Taïwan, renonçant à contrôler les « invisibles » (c'est-à-dire les transferts monétaires), a concentré ses efforts, d'une façon très efficace, sur le 
contrôle de l'immigration illégale et de l'espionnage. Enfin, les États d'Asie orientale ont été renforcés dans leur indépendance par les événements extérieurs. En Asie comme ailleurs, la fin de la guerre froide a provoqué la disparition des grands alignements politiques et donc à la fois le départ des troupes soviétiques du Vietnam et la réduction des troupes américaines stationnées au Japon et en Corée du Sud. Les États-Unis se trouvent, là aussi, dans la situation de rester la seule puissance mondiale, mais de n'avoir ni les moyens, ni la volonté, ni les conceptions nécessaires à l'exercice d'un leadership clair. Elle est aggravée, dans la région, par un certain nombre de difficultés spécifiques, suivant les cas commerciales ou politiques, qui confèrent une allure hésitante et oscillatoire aux rapports de Washington avec le Japon, l'Inde, et surtout la Chine. Le résultat est que les différents États asiatiques disposent de moyens de manoeuvre beaucoup plus vastes. C'est ce que les journaux de la région désignent avec inquiétude comme le "vide stratégique » de l'Asie. Des efforts ont été accomplis, depuis quelques années, sinon pour le combler, du moins pour réduire ses inconvénients : mise en place d'une "conférence post-ministérielle » à laquelle sont conviés les principaux partenaires de l'ASEAN ; et organisation, par la même ASEAN, à partir de 1994, d'un «Forum asiatique régional » qui discute des grands problèmes stratégiques régionaux (en 1995, le conflit sur les Spratleys et les essais nucléaires français). Le progrès est réel, mais limité, notamment parce que les grandes puissances d'Asie du Nord-Est (Chine, Japon) n'ont aucune intention de donner aux petits pays d'Asie du Sud-Est un quelconque droit de regard sur leur politique extérieure. L'évolution des deux dernières décennies conduit donc à l'idée d'un contraste croissant entre les faits économiques et politiques. Dans le domaine économique, ce qui est moteur, c'est la régionalisation. Dans le domaine politique, c'est le renforcement du pouvoir des États-nations. Là est sans doute le paradoxe central de l'Asie orientale contemporaine. Il faut comprendre comment fonctionne le couple apparemment inversé des forces économiques et politiques. Car ce ne sont pas des forces totalement indépendantes. Elles contribuent à se façonner réciproquement. La façon dont le politique contrôle et libère à la fois l'économique est justement expliquée par les thèses sur l'«État riziculteur»: le politique prépare, surveille, assure l'économie, mais respecte son autonomie ${ }^{5}$. C'est au Japon puis dans les Nouveaux pays industrialisés que cette pratique a été expérimentée. Elle n'est pas sans lien avec le paternalisme confucéen, très présent dans ces sociétés. L'État-père guide, protège et surveille les pas des acteurs économiques. De leur côté, ceux-ci s'interdisent d'intervenir dans son domaine d'élection - les grandes affaires du pays - et concourent pratiquement, chaque fois que nécessaire, à l'application de ses décisions. Dans de nombreux cas, nous l'avons $\mathrm{vu}, \mathrm{c}^{\prime}$ est bien ce qui s'est produit.

5 La crise du politique Ce partage des responsabilités, envisagé rétrospectivement, semble simple et pratique. On lui attribue volontiers un rôle important dans les succès économiques du Japon et des NPI. En pratique, il s'est installé, difficilement, et n'a diffusé que tardivement dans le reste de la région. Le fait est, pourtant, qu'une fois installé il a concouru à réduire le champ du politique comme instance de choix, donc d'hésitation et de trouble. Au Japon depuis le début des années cinquante, et dans les NPI depuis le début de la décennie suivante, les gouvernements étaient stables, leurs politiques durables, la seule actualité paraissait celle de l'économie. Seules quelques turbulences, d'ailleurs souvent tragiques, ont agité une trentaine d'années de discipline et de progrès: elles ont été rapidement dominées. En fait, élites et populations s'accordaient sur un petit nombre d'objectifs : pousser la production pour hausser les 
conditions de vie, moderniser la société tout en conservant le maximum des disciplines héritées de la tradition, augmenter les moyens de l'indépendance en profitant de la protection américaine. Ce schéma, qui n'a jamais été parfait, s'est progressivement imposé comme le schéma de base des systèmes politiques asiatiques: c'est lui qui commande le modèle à la fois autoritaire et dévelopementaliste vers lequel ont évolué les pays d'Asie du Sud-Est (Indonésie, Malaisie, Thaillande et aujourd'hui peut-être Philippines) puis les systèmes communistes (Chine, Vietnam). Cette extension géographique n'a cependant pas entraîné de succès complets : ces deux catégories de pays connaissaient des problèmes spécifiques dont ils ne pouvaient se débarrasser d'un coup. Pour ne prendre qu'un exemple, deux systèmes politiques asiatiques se trouvent actuellement sous la menace d'une difficile crise de succession : ceux de Chine et plus tard d'Indonésie. Surtout, ce schéma général a été brouillé, à partir du milieu des années quatre-vingt, par deux grandes mutations, l'une internationale, et l'autre sociale. L'érosion, puis la fin de la guerre froide, ont libéré des processus de mondialisation et de régionalisation qui ont menacé le délicat équilibre entre fermeture et ouverture que le Japon et ses disciples avaient échafaudé. Pour résumer, disons que cet échafaudage reposait sur un compromis de nature variable entre fermeture politique et culturelle (plus forte au Japon) et ouverture économique (moins accentuée dans le même Japon). Mais, en Asie comme ailleurs, la mondialisation n'a pas seulement agrandi les ouvertures économiques, elle a aussi attaqué les centres nerveux qui commandaient les politiques et les perceptions d'indépendance. La régionalisation de l'Asie - c'est toute son ambiguïté - a été à la fois un effet de cette ouverture accentuée et aussi, nous l'avons vu, un effort à la fois individuel et collectif pour la réduire au domaine de l'économie. Il n'en reste pas moins, cependant, qu'en Asie comme dans le reste du monde, le droit d'un État-nation à édifier un système politique différent de la vulgate démocratique diffusée par l'Occident fait aujourd'hui problème. Les États d'Asie ont beaucoup plus de mal qu'avant à cacher leurs crimes, voire leurs peccadilles. Alors que les généraux indonésiens avaient pu massacrer en toute impunité des centaines de milliers de communistes en 1965-1966, les dirigeants chinois ont dû admettre les télévisions étrangères sur la place Tiananmen en 1989, et les Singapouriens s'étonnent aujourd'hui de devoir justifier à la face du monde un arsenal de punitions qui paraît médiéval en Occident. En tout cas, l'effet général de ces arrestations est que la politique autoritaire des nouveaux pouvoirs asiatiques rencontre un problème général de légitimité. L'autre grande mutation affecte les politiques intérieures, et elle dérive des succès mêmes de l'économie. Non seulement il y a de plus en plus d'argent dans les sociétés est-asiatiques, mais les États ont conservé ou renforcé leurs leviers de contrôle : le résultat est que la corruption politique qui a une longue histoire dans cette région du monde n'a cessé de s'étendre et de s'aggraver. Présente dans tous les systèmes, y compris les plus moralistes, elle gangrène la plupart des centres de décision politiques. Elle sape la réputation et l'autorité des pouvoirs. Ceux-ci doivent de plus en plus répondre à un soupçon universel, soit par un surcroît de moralisme officiel, soit en changeant leurs titulaires. Mais cette réaction n'a pas pu, au Japon du moins, réduire la crise civique engendrée par deux décennies de corruption à ciel ouvert. En même temps, l'autorité des pouvoirs est atteinte par une deuxième conséquence sociale du succès économique: la modification des rapports entre les individus et les collectivités. Pour résumer - car les nuances et les variations ont été innombrables - rappelons qu'en Asie orientale les collectivités familiales, territoriales et économiques ont conservé très tardivement un pouvoir considérable sur leurs 
membres. De là, dans la plupart des pays, une cohésion sociale cellulaire que les pouvoirs politiques ont plus ou moins habilement manipulée pour assurer leur contrôle et renforcer la discipline du travail. Mais, comme on pouvait le prévoir, l'élévation considérable des niveaux de vie et l'entrée de plusieurs pays (Japon, Corée du Sud, Taïwan, Singapour) dans la société de consommation ont complètement changé le statut social de l'individu. Les pays plus retardés abritent eux aussi des transitions qui vont dans le même sens. Le résultat est que, dans toute la région, l'espace privé de l'individu s'élargit et les liens qui l'attachent aux collectivités perdent de leur évidence. Les morales traditionnelles - notamment confucéenne - sont affaiblies et la question des valeurs morales est agitée partout: les sociétés asiatiques doivent-elles se laisser évoluer vers un individualisme de type occidental ou réinventer des «valeurs asiatiques ", voire forcer autoritairement leur application ? Une autre conséquence de cette poussée de l'individu est l'élévation des exigences qu'il adresse à tous les pouvoirs, économiques et politiques : ce qui est aujourd'hui spectaculaire au Japon, à Taïwan et en Corée, est manifeste en Thaïlande et en Chine, et même perceptible à Singapour ou en Indonésie. Tous les systèmes autoritaires de la région sont donc confrontés, à des degrés divers, à des aspirations qui ne trouvent pas toujours d'expression "politique » ou "partidaire ", mais qui impliquent toutes un plus grand respect matériel et spirituel de l'individu ${ }^{6}$. Ainsi, l'ensemble de la région est traversé par une crise générale du politique. Cette crise est cachée de plusieurs façons : par les propagandes et les conformismes des régions, par le brouhaha des échanges économiques, mais surtout par la hausse générale des revenus et l'amélioration des conditions de vie, qui facilitent les répressions et les replâtrages auxquels les pouvoirs procèdent régulièrement. Les sociétés asiatiques ne sont pas amorphes, elles ne cessent de bouger, de revendiquer, d'aspirer à plus d'espaces d'autonomie. Mais, à tort ou à raison, elles exercent une sorte de sagesse empirique qui consiste à engranger les bienfaits matériels de la croissance et, quand la rébellion ne " paye " pas, à poursuivre, sous les apparences de la discipline, un travail de sape continuel. De ce point de vue, l'élévation du taux de divorce à Singapour est aussi significative que les migrations de population vers les zones côtières en Chine. De leur côté, les pouvoirs ne se contentent pas de colmater et de calfeutrer, ils évoluent sans arrêt: les uns, vers leur propre version de la démocratie; d'autres vers un regain de moralisme autoritaire ; d'autres encore tâtonnent.

6 La poussée des nationalismes La crise du politique en Asie orientale est dangereuse car, dans un cadre régional de rapprochement économique mais dénué d'organisation claire, les crises intérieures risquent de réactiver un certain nombre de fractures anciennes et de leur en ajouter de nouvelles ${ }^{7}$. Rappelons un certain nombre de banalités bien souvent oubliées. Tout d'abord, qu'au lendemain de la Deuxième guerre Mondiale l'éclosion des nationalismes a pris une forme particulièrement intense dans la majeure partie de l'Asie orientale. ${ }^{8}$ En second lieu, que les conflits attisés par la guerre froide y ont atteint une ampleur et une violence effroyables, et pas seulement en Indochine : on a bien tort aujourd'hui d'oublier que la Malaisie était à feu et à sang à la fin des années quarante, que la guerre de Corée a mis en danger la paix du monde en 1950-1951, que l'Indonésie a failli glisser vers le communisme avant le coup d'État sanglant de septembre 1965, et que Bangkok était encore menacée par la guérilla à la fin de la décennie suivante. Ces drames ont laissé des traces profondes et vivaces dans les mémoires. Ils s'ajoutent à bien d'autres mauvais souvenirs, les uns très anciens (par exemple les rancoeurs des anciens tributaires de la Chine comme le Vietnam, la 
Mongolie ou la Corée), et les autres qui remontent aux guerres de la première partie du siècle, et notamment aux atrocités commises par les Japonais en 1937-1945. Nombre de conflits se sont exprimés dans des conquêtes, ou des partitions, ou des contestations de frontières qui ont laissé des rancoeurs, des irrédentismes et des haines. Bien souvent, les progrès économiques et la circulation des biens ont recouvert ces brandons de discordes sans les éteindre. Les mutations récentes ont apporté deux éléments nouveaux. Le premier, c'est l'enrichissement qui a élevé les enjeux de la politique régionale: il est beaucoup d'immenses fortunes, privées ou publiques, dans l'Asie d'aujourd'hui. Or, non seulement la richesse est inégalement répartie, mais elle est plus ou moins disjointe de la puissance militaire : les pays les plus riches sont en général les moins bien armés (Singapour) ou surtout les moins aptes politiquement à se défendre (Japon, Taïwan); les plus pauvres sont au contraire très armés (la Chine) ou comparativement mieux armés (Indonésie, Vietnam). Les résultats de cette inégale répartition de la richesse et de la force sont la course aux armements chez les plus riches et la banalisation de la menace et du chantage chez les plus forts, en particulier la Chine. Le deuxième élément nouveau, c'est que la régionalisation économique pose de façon de plus en plus insistante la question d'une organisation régionale. On lit ici ou là que les Asiatiques seraient culturellement rétifs aux contrats et aux institutions de nature collective. Faut-il rappeler que, durant la guerre froide, l'Asie a eu son lot de traités, d'alliances et de coalitions? Ce qui est plus vrai, c'est que les gouvernements de la région sont devenus avant tout pragmatiques. Pour la majorité d'entre eux (mais pas la Malaisie), une organisation économique de l'Asie orientale présenterait entre autres inconvénients celui de poser un problème insoluble de hiérarchie entre les principales puissances de la région, notamment entre la Chine et le Japon. C'est pourquoi ils ont accepté, à titre transitoire, le développement de l'APEC, qui ne règle ni ne gâche rien. A défaut d'être organisée ou admise, la compétition a été retardée. Mais elle reste inévitable. Or, tout donne à penser par ailleurs que le nationalisme apparaît de plus en plus comme la seule solution évidente aux crises politiques intérieures. D'abord, parce que les solutions alternatives paraissent inefficaces ou incomplètes. Peut-être est-ce en partie pour freiner son émergence que les autorités singapouriennes et malaisiennes, qui sont les plus "cybernétiques" de la région, ont prôné, depuis le début de la décennie, la supériorité de supposées « valeurs asiatiques » sur les valeurs occidentales. Elles ont ainsi proposé au reste de l'Asie orientale une véritable plate-forme commune, à la fois morale, idéologique et politique : vertu privée, discipline publique, priorité à la croissance économique. A plusieurs reprises, ce discours semble avoir fait des émules dans les autres gouvernements asiatiques, soit parce qu'il légitimait leur autorité, soit encore (et surtout), parce qu'il fournissait une argumentation facile pour une résistance aux intrusions de l'Occident en matière de droits de l'homme, de réglementation économique ou d'environnement. Par ailleurs, ici ou là, notamment en Chine et au Japon, des intellectuels s'efforcent de fonder sur les débris du néoconfucianisme les lignes de forces d'une résistance philosophique à la mondialisation. Il faudra donc examiner de près, dans les années prochaines, l'évolution du débat idéologique qui a été déclenché à Singapour et Kuala Lumpur. En attendant, on doit mettre en évidence les faiblesses congénitales des "valeurs asiatiques». Celles-ci, en effet, n'amalgament aucune des transformations qui, d'ores et déjà, s'étendent de facto dans toutes les villes asiatiques: la libéralisation des moeurs, le goût du loisir, l'individualisation des comportements. De plus, elles sont portées par des responsables gouvernementaux, et non par des élites sociales modernes, ce qui affaiblit 
inévitablement leur attraction. $\mathrm{Si}$, comme il est probable, les «valeurs asiatiques » ne parviennent pas à s'imposer comme une réponse globale à la crise, quelles sont les autres possibilités? Le marxisme, en Asie orientale comme ailleurs, a pratiquement disparu de la scène idéologique. La religion n'est pas considérée comme capable de porter un projet de société - sauf dans les pays musulmans où diverses formes d'islams politiques se développent, mais freinés par des pouvoirs civils méfiants. La démocratie paraît certes inscrite dans l'horizon: à cause de ses connexions avec un libéralisme économique que personne ne conteste et aussi parce qu'elle paraît répondre aux exigences nouvelles des individus. Aux yeux des élites asiatiques, la démocratie est une caractéristique de la modernité. Mais elle ne constitue ni un absolu, ni un idéal clos. C'est un cadre institutionnel qu'il faut mettre en place quand cela s'avère nécessaire et possible : des impératifs économiques ou la nécessité de protéger la stabilité politique peuvent justifier des retards. De plus, la démocratie ne fournit pas le "contenu" d'un modèle parce qu'elle ne répond pas aux grands problèmes moraux et ne garantit pas la discipline sociale. Sur le "marché des modèles ", il ne reste donc que le nationalisme pour combler un vide de plus en plus béant. Car lui seul, en Asie orientale comme dans bien d'autres lieux, peut prétendre à la fois légitimer le pouvoir, fonder la cohésion, sociale et politique, justifier la mise en place de valeurs spécifiques, et donner forme au désir de défendre ou d'acquérir des richesses ou du statut. L'évolution paraît déjà en cours, même si elle rencontre ici ou là des obstacles : par exemple, les souvenirs de la deuxième guerre mondiale rendent difficile une "sortie de crise" nationaliste au Japon. Au Vietnam, le régime communiste défaillant a déjà tant usé du ressort nationaliste que la population, recrue d'épreuves, regarde provisoirement dans une autre direction; à Taïwan, la menace chinoise et les divisions intérieures rendent dangereux le recours à une thématique "indépendantiste ». Ailleurs, en revanche, le ressort national est déjà dressé. Le cas de la Corée du Sud est particulier, puisque la menace du Nord contraint à une surenchère nationaliste $:$ la haine du Japon y constitue presque un alphabet commun. Dans les pays d'Asie du Sud-Est, le nationalisme est à vif, à l'état spontané et éventuellement anti-gouvernemental comme aux Philippines (où il a récemment explosé contre le voisin singapourien), voire contre des voisins (Thaïlande) ou contre une dissidence (Timor oriental pour l'Indonésie). En Chine, le nationalisme ne cesse de monter depuis les événements du printemps 1989 car il est attisé par des autorités soucieuses de remédier à l'évidement idéologique du communisme. La politique le plus souvent brutale en direction de Hong Kong et des îles de la mer de Chine du Sud, les défis que Pékin multiplie à l'encontre de la superpuissance américaine et les discours agressifs en direction du Japon méritent d'être interprétés en large partie de cette façon. Le nationalisme - les nationalismes - d'Asie orientale pose bien des questions qu'il est trop tôt pour discuter, tant sont grandes et volatiles ses différences avec celui qui prévalait au lendemain de la deuxième guerre mondiale. Il était alors massif, populaire, ouvert sur des causes plus larges, et donc capable d'inspirer des combats longs et difficiles contre le principal ennemi, l'Occident colonialiste ou impérialiste. Aujourd'hui, les réactions nationalistes sont moins prévisibles et moins construites, socialement moins centrales et d'expression moins continue. Elles ne sont en général plus conduites par des partis ou des mouvements de masse, mais sont plus souvent soit spontanées, soit manipulées par les gouvernements. Une raison de ce changement est que leur cible n'est plus aussi évidente. C'était autrefois l'Occident, que chacun jugeait responsable du sous-développement et de l'abaissement national. Or, tout en continuant d'agacer les susceptibilités nationales, 
l'Occident a cessé de poser un problème politique majeur en Asie, et personne dans la région ne conteste qu'il demeure un partenaire économique, voire stratégique, indispensable: les nationalismes d'Asie se développent sur fond de mondialisation. C'est donc vers les voisins asiatiques que les nationalismes se tournent, ces mêmes voisins dont la régionalisation fait en même temps des partenaires. Le processus n'en est certes qu'à ses débuts. Il est voilé par les banderoles triomphales du progrès économique, et parfois rejeté au second rang par des heurts plus immédiats avec des puissances extérieures à la région, et d'abord les États-Unis. Il rencontrera de puissants obstacles, notamment dans les milieux d'affaires, partout très influents. Mais il est déjà à l'oeuvre, le plus visiblement sur les marges du pays le plus instable qui occupe en même temps une position géographique centrale, la Chine : Hong Kong, Taïwan et les îles Spratleys figurent, sur la carte actuelle de l'Asie orientale, comme des clignotants annonciateurs d'un avenir beaucoup moins facile. Derrière l'Asie orientale marchande qui est tantôt célébrée et tantôt crainte, il faut donc mettre en évidence un champ à la fois moins visible et moins vu, celui de la politique régionale. D'abord, pour montrer que la régionalisation économique de l'Asie orientale est elle-même l'effet d'un processus dans lequel la politique a joué un rôle. Ensuite, pour indiquer que la période du régionalisme économique triomphant touche sans doute à sa fin. La croissance des PNB et les mutations sociales engendrent aujourd'hui une crise politique dont l'issue pourrait bien être une intensification des rivalités nationales dans la région.

\section{NOTES}

1. Nous entendons par Asie orientale, l'addition de l'Extrême-Orient et de l'Asie du SudEst. L'ASEAN et son expression toujours programmée l'AFTA (Asia Free Trade Association) comprend sept pays d'Asie du Sud-Est : Indonésie, Malaisie, Singapour, Philippines, Thaïlande, Brunei et, depuis 1995, le Vietnam. L'Asian Pacific Economic Cooperation regroupe les principaux pays des deux bords du Pacifique, États-Unis compris.

2. Les pages qui suivent peuvent être considérées comme une discussion de l'ouvrage brillant mais à notre sens excessif de Bertrand Badie, sur La fin des territoires, qui surévalue l'importance et les effets des flux transnationaux, au moins en Asie orientale. 3. Chen Jie, «Human Rights : ASEAN's New Importance to China », The Pacific Review, Vol. VI, $n^{\circ}$ 3, pp. 227-237.

4. Sur la question des « valeurs asiatiques » et plus généralement d'une « idéologie de l'Asie », cf. David Camroux et Jean-Luc Domenach, Vers une réinvention de l'Asie ?, Le Seuil, à paraître.

5. Voir la contribution de Pierre Judet à l'ouvrage du GEMDEV sur L'intégration régionale dans le monde, innovations et ruptures, Paris, Karthala, 1994.

6. Sur ce point, nous nous rapprochons de certaines analyses de Robert A. Scalapino. Voir par exemple The Politics of Development, Perspectives on 20th Century Asia, Harvard University Press, 1989. 
7. Sur les problèmes de sécurité en Asie, le travail de base est celui de Barry Buzan et Gerald Segal, « Rethinking East Asian Security », Survival, été 1994, pp. 3-21.

8. Sur ce point (comme sur d'autres, d'ailleurs), on se reportera avec fruit au livre de François Godement, La renaissance de l'Asie, Odile Jacob, 1993.

INDEX

Mots-clés : politique

Index géographique : Asie du Sud-Est, Chine 